\title{
TYPOLOGIE DES FORMULES MAGIQUES
}

\section{Claude Lecouteux}

This paper provides information on magical formulas in both medieval and nearcontemporary charms, based on a corpus of more than 1000 formulas, and tries to give a typology.

Key words: oral and written formulas; pagan formulas; Christian formulas; vocabulary of the formulas

Une formule magique est un ensemble de mots et/ou de signes permettant de réaliser l'irréalisable en faisant appel à des forces surnaturelles représentées par Dieu, les dieux, les représentants de la foi chrétienne (apôtres, saints, etc.), les démons ${ }^{1}$, les anges planétaires ou décaniques (Lecouteux 2004 : 167 passim) et les esprits. Au XVI ${ }^{\mathrm{e}}$ siècle, en Angleterre, on invoque même les fées (Scott 1584 : XV, 10).

La formule convoque ces puissances numineuses et les force à agir dans le sens voulu. La convocation, plus exactement la « citation ", est un ordre auquel ils ne peuvent se soustraire, car le verbe est tout puissant. Si l'on sait à qui s'adresser, si l'on possède un nom, la formule est encore plus efficace. Mais certains mots isolés peuvent posséder autant de force qu'une formule, et il est notable que le vocable magique se rencontre souvent au début ou à la fin des charmes, revêtant la valeur d'un impératif, au même titre que l'omniprésent fiat, répété trois fois.

Les formules reposent sur des assonances (kalepiis avalapiis), des allitérations (max, nax, pax), des anaphores (magula magulus), des répétitions et des variations (ala drabra ladra dabra rabra afra brara agla et alpha omega) ${ }^{2}$; elles font un large usage de l'homéotéleute (mala magubula). Tout cela leur confère un aspect rythmé, mélodique, celui d'une psalmodie, d'une mélopée, ce que rendent les verbes incantare et incarminare, le norrois runa et l'allemand raunen. En norrois, le charme se dit galdr (ags. gealdor ${ }^{3}$ ), sur le verbe gala, " chanter $^{4}$ ", et en vieux haut-allemand, un des termes est galdar / galster ${ }^{5}$; dans cet idiome, nous avons bigalan, " ensorceler, enchanter », littéralement « chanter près de »(Wesche 1940 : 40 sqq.). 
Le rythme produit par les moyens linguistiques cités me semble déterminant. Du reste, dans la description d'une séance de nécromancie en Islande au $\mathrm{XI}^{\mathrm{e}}-\mathrm{XII}{ }^{\mathrm{e}}$ siècle, l'accent est mis sur la diction, et tout cela rappelle les rituels chamaniques (Gravier 1955 : chap. 4). Les charmes grecs déjà vocalisent ainsi par de longues séries de voyelles ${ }^{\mathbf{6}}$.

Les formules se sont essentiellement transmises par le canal de l'écriture, ce qui ressort, inter alia, du mélange de caractères latins et grecs, ce qu'illustrent des charmes anglais (Storms $1948: \mathrm{n}^{\circ} 32 ; 59$ ) et scandinaves, parfois hébraïques (Lecouteux 2005 : 128 ; 131), comme dans un sachet accoucheur auvergnat, et des runes (Beckers 1984 ; Bang 2005 : n 1085 et 1128 ; Saemundsson 1992 : $n^{\circ} 18,27$ et 44$)$. Des erreurs de lecture et de transcription les ont rendues inintelligibles. En outre, celles qui nous sont parvenues témoignent, du moins pour les formules chrétiennes et mixtes, d'une interaction permanente entre écrit et oral. Certains charmes attestent des transcriptions d'ordre quasi phonétique, et nous pouvons nous demander si un auditeur n'a pas tenté de s'approprier le charme récité et sa formule. On sait que les ménagers et autres livres domestiques ont compilé toutes sortes de recettes, dont des charmes, sanitaires et médicaux le plus souvent, le Mesnagier de Paris (Le Mesnagier 1994 : 471, 789) et le Hausbuch de Michael de Leone ${ }^{7}$ l'attestent.

Les caractéristiques essentielles des formules non chrétiennes sont leur obscurité. Elles mêlent des mots de diverses langues et, pour être magiques, il suffit qu'ils appartiennent à un idiome étranger à l'opérateur. L'allemand devient ainsi magique dans quelques charmes scandinaves (Bang 2005 : $\mathrm{n}^{\circ}$ 1085 ; Ohrt $1921: \mathrm{n}^{\circ}$ 821). La diversité des langues de ces noms s'explique si l'on se souvient du mot d'Origène (vers 185-253) : "Les noms qui possèdent une puissance dans une langue, la perdent lorsqu'ils sont traduits " (Contre Celse I, 45).

Quel que soit le type de charme, le latin y joue un rôle primordial. Il est d'autant plus magique qu'il est défiguré au point d'en devenir incompréhensible, semblant avoir été transcrit phonétiquement. Le Credo et le Pater noster, tout ou fragment, se transforment ainsi en formules magiques. Sur une amulette du

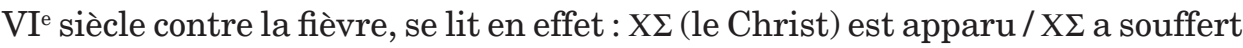
/ X $\Sigma$ est mort / X $\Sigma$ est ressuscité / X $\Sigma$ est enlevé (au ciel)... (Boswinkel - Pestman 1978 : 98), ce qui est une partie du Credo ; en Norvège, nous avons : Kredor nijande potamen (Bang $2005: \mathrm{n}^{\circ}$ 1337c). Mais le grec donne quelques formules simples comme $\pi \alpha \rho \alpha \pi \alpha \rho \alpha \pi \alpha \rho \alpha$ dans un charme danois (Ohrt 1921 : II, 120), ou des mots qui s'insèrent dans les charmes (Panthon, Diaton, usion, on, etc.).

D'autres formules sont des fragments des ces prières écrites à l'envers. Pour transformer une prière en formule magique, il suffit de la réciter à rebours lors de l'opération, procédé encore utilisé en Islande au XIX ${ }^{\mathrm{e}}$ siècle. 
Je laisserai de côté la magie dite cérémonielle, qui est représentée par la tradition salomonienne, Clavicula maior et minor, Liber iuratus, Ars notoria ${ }^{8}$, etc., car les formules prennent la forme de longues oraisons faites de dizaines de mots à consonance grecque, hébraïque et latine.

Les formules se répartissent en deux grandes familles : celles pouvant être dites et celles qui ne le peuvent. Dans la première, nous pouvons, cum grano salis, distinguer 3 types.

\section{FORMULES ORALES}

1. Les formules chrétiennes tirées de la Bible et de la liturgie ; elles sont communes à toute l'Europe occidentale et septentrionale. On les trouve dans les oraisons magiques, la iatromagie et les charmes. En voici des exemples :

Christus vincit, Christus regnat, Christus imperat ${ }^{9}$

Jesus autem transiens (Luc 4, 30)

Crux Christi reducat ${ }^{11}$

Christus natus, Christus passus, Christus cruxifixus, Christus mortuus ${ }^{12}$

Os non comminuetis ex eo (Jean 19,36), censée guérir le mal de dent

(Ohrt $1921: \mathrm{n}^{\circ} 443$ )

Dans ce type, nous avons des formules cumulatives, composées d'épiclèses noms, épithètes et d'attributs - du personnage invoqué ; ainsi on met en œuvre sa virtus, car nomen est numen. Les noms de Dieu, etc., sont énumérés, comme dans les litanies. On en trouve un bon exemple dans le Bref du Pape Léon, adressé à Charlemagne, et dans les oraisons et charmes faisant appel aux 72 noms ${ }^{13}$. Notons qu'il faut les écrire avec des croix, comme le précise un manuscrit : ista nomina scribantur cum cruce (Hunt 1994 : 348).

2. Les formules païennes, dont une partie s'est transmise par Marcellus de Bordeaux, les Cyranides, le Picatrix ${ }^{14}$, plus généralement, par les codex de médecine et de pharmacie, et même par des traités d'agriculture, comme celui de Caton l'Ancien (234-149 av. J. C.) où nous rencontrons Motas Vaeta Daries Dardares Astataries Dissunapites, dont on retrouve des fragments aux XVI ${ }^{\mathrm{e}}$ et XVII ${ }^{\mathrm{e}}$ siècles sous la forme : Danata Daries Dardaries Astraries ${ }^{15}$. Notons que dès le $\mathrm{XI}^{\text {e }}$ siècle l'influence de traités médicaux d'origine grecque se fait sentir en Angleterre dans le Leecebok et les Lacnunga ${ }^{16}$. Des fragments de prières grecques incomprises, comme Stomen calcos (Singer 1917) et Eulogumen patera (Franz 1909 : II, 578 ; Storms 1948 : n²61), deviennent des formules magiques païennes. 
3. Les formules mixtes où des mots magiques s'introduisent dans les formules chrétiennes et les renforcent. C'est le cas d'Agla, de Sator Arepo. Ce type a occupé une place importante dans les traditions populaires.

\section{FORMULES ÉCRITES}

La deuxième famille est la plus diversifiée et la plus mystérieuse, car elle se compose de lettres, de signes appelés caracteres, nomina barbara et ephesia grammata (Ogden 1999 : 47 ; Peterson 1921 ; Bohak 2003) - les Grecs rapprochèrent ephesia d'une inscription du temple d'Artémis à Ephèse alors que, sans doute, le vocable vient du babylonien epêšu qui signifie " envoûtement. » Cette famille forme le gros des inscriptions des amulettes et phylactères, qui se rencontrent en grand nombre dans les papyri magiques grecs. On peut supposer que les formules composées de lettres résument l'essence d'un charme, en sont le noyau dur, dont la forme a été spécialement choisie pour tenir sur une petite amulette faite de tous les supports possibles : parchemin et papier, bois et métal - dont le plomb, comme les tablettes de défixion de l'Antiquité classique -, hostie, pomme, etc. Il n'est pas impossible que les successions de lettres aient possédé une fonction mnémotechnique, rappelant à l'opérateur la formule à prononcer. La présence d'abréviations latines qui se rencontrent d'habitude dans les manuscrits médiévaux parle en faveur de cette hypothèse.

Nous en distinguons 8 types :

1. Les successions de lettres qui sont parfois l'initiale de versets ou de passages de la Bible, à l'image du Psaume 119 et des Proverbes 31, 10 sq. La Bénédiction de Zacharie (Gompert 1918) et celle de saint Benoît ${ }^{17}$ en sont une belle illustration. Les paroles du Christ (Luc 23, 46) Pater in manus tuas commendo spiritum meum son notées PIMTCSM ; qui ne connaît le Nouveau Testament par cœur n'en devinera jamais le sens.

2. Les abréviations, comme le tétragramme (IHVH), le titulus triumphalis du Christ (INRI), le monogramme du Christ (IHS), les noms des rois mages (CMB) (Wackernagel $1876: 611$ ), consummatum est $(=\mathrm{CE})^{18}$, et celles, courantes de formules de prières ${ }^{19}$. Le charme de sainte Agathe s'écrit ainsi : MSDHDEPL (=Mens sancta, Spontaneus honor Dei et Patria Liberatio).

3. Les mots tronqués, dont on ne donne que la première syllabe ou dont on omet les voyelles, ou des mots, des prières à l'envers (Árnason 1862-1864 : I, 317 sqq.). Dans une conjuration contre un voleur, on lit : Dnaa. Gilleh. Go Nøs, Redaf Dug (Bang 2005 : n 1377), c'est-à-dire : "gud fader, søn og hellig aand » (Dieu le père, le Fils et le saint Esprit). 
4. Les lettres initiales de vocables forment parfois un nouveau mot qui condense toute une phrase ; c'est le cas d'Agla (Lecouteux 2001), d'Ananizepta (Seligman 1921) et d'Ablanathanalba (PGM XXXIII, 1-25 ; XXXVI, 211-30), de Jebela (= Job der lak in dem miste, "Job gisait sur le fumier ») (Anzeiger : $\left.279, \mathrm{n}^{\circ} 3\right)$.

6. Les lettres formant un mot dépourvu de sens, utilisé comme formule réductrice en magie sympathique ou analogique : en en retranchant les lettres une à une, on réduit la maladie que l'on veut guérir. Le mot le plus connu est Abracadabra (Deonna 1944), parfois crypté en remplaçant les A par des H (Albertus Magnus : 1, 222), Marcellus connaît Sicycuma ${ }^{\mathbf{2 0}}$, mais il en existe bien d'autres, comme Calamaris (Ohrt 1921 : II, 110-112 ; Espeland 1974 : n 28) et Oipilu (Bang $2005: \mathrm{n}^{\circ}$ 1056) en Scandinavie.

À l'inverse, les formules croissantes sont rares.

7. Viennent les signes. Ils sont empruntés à des alphabets forgés sur le grec et l'hébreu, comme ceux attribués à Apollonius de Tyane ${ }^{21}$, ou à des alphabets dont les lettres sont calquées sur les constellations du ciel, comme l'écriture céleste et celle dite de Malachim ${ }^{22}$, et les deux alphabets de Salomon ${ }^{23}$ par exemple. On les rencontre principalement dans des traités traduits de l'arabe en Espagne dans la seconde moitié du XIII ${ }^{\mathrm{e}}$ siècle. Jusqu'au $\mathrm{XVI}^{\mathrm{e}}$ siècle, ces alphabets sont à peu près stables, puis on en rencontre de nouveaux, la charnière étant représentée par la Stéganographie de l'abbé Trittheim.

8. Les formules mêlant lettres et signes ${ }^{24}$.

Pour conclure, je soulignerai un point qui me semble important. Les charmes d'Europe centrale et orientale comportent peu de formules à ma connaissance. Emanuela Timotin en a relevé deux dans ses travaux (Timotin 2009). Pourquoi en est-il ainsi ? La religion a dû jouer un rôle déterminant. Ce ne serait guère étonnant car ce sont en grande majorité les clercs qui ont recopié et diffusé les charmes. Jusqu'au XIX ${ }^{\mathrm{e}}$ siècle, on les tint pour des magiciens, Agobard de Lyon (778-840) y fait déjà allusions, les pénitentiels blâment les prêtres qui utilisent les charmes et font des amulettes ${ }^{25}$, et les traditions populaires le confirment ${ }^{26}$. En outre, la part très importante que prennent Bible et liturgie dans les charmes et dans la constitution de formules et oraisons dont la structure décalque celle des prières chrétiennes ouvre une intéressante perspective, celle de la religion comprise comme magie. Il faut donc considérer ce complexe en athée, position indispensable si on ne veut méconnaître la mentalité sous-jacente aux charmes. Dès lors, le christianisme ressort comme une religion à mystère illustrée par la messe et la transsubstantiation, voire par les litanies. Il est donc normal d'en utiliser les éléments pour obtenir ce que l'on demande, ce qui n'empêche pas 
de se tourner en même temps vers les anciens dieux (Saemundsson $1992: \mathrm{n}^{\circ}$ 33 et 44) ! Dans un charme islandais utilisant une rune non identifiée, se lit :

Ægishjálm er eg ber á millum augna mér. Reiðin renni, stríð stemmi. Verði mér svo hvör maður feginn sem María varð fegin sínu[m] signuðum syni pá hún fann hann á sigurhellunni. Í nafni föður og sonar og anda heilags

Je porte un heaume de terreur (AEgishjálmr) ${ }^{27}$ entre mes yeux. Colère disparais, inimitié arrête ! Que chaque homme devienne bon envers moi comme Maria fut bonne envers son fils bénit quand elle l'a trouvé sur la dalle de victoire. Au nom du Père et du Fils et du Saint-Esprit. (Saemundsson $1992: \mathrm{n}^{\circ}$ 41)

Bel exemple de mixte pagano-chrétien !

\section{NOTES}

1 Cf. le Testament de Salomon dans Lecouteux 2005 : 66-70.

2 van Haver $1964: n^{\circ} 270$.

3 En anglo-saxon, le terme entre dans des composés avec les vocables « mot » et « chant » (gealdorword, wordgealdor; gealdorleod, gealdorsang).

4 L’adjectif galinn signifie " enchanté, hors de sens ».

5 Les autres étant garminon et spell.

${ }^{6}$ Cf. par exemple Bonner $1950: \mathrm{n}^{\circ} 2$.

7 Munich, Universitätsbibliothek, $2^{\circ}$ Cod. Ms. 731 (XV ${ }^{\mathrm{e}}$ siècle), fol. 211r ${ }^{\circ}-225 v^{\circ}$.

8 Véronèse 2007 ; Ars notoria 1657 ; Hedegård 2002.

9 Franz 1909 : II, 87 ; 96 ; 106 ; 49 ; Ohrt $1921: \mathrm{n}^{\circ} 266$; 269 ; 329 sq. ; 1087 ; 1144 ; 1156 ; Braekman $1997: \mathrm{n}^{\circ} 49 ; 73 ; 101 ; 148 ; 151$ sq. ; $164 ; 234$.

${ }^{10}$ Braekman $1997: \mathrm{n}^{\circ} 267$; 272 ; 277 ; Hunt 1994 : 94 ; Ohrt 1921 : n 230 ; Arnaldus de Villanova fol. $302 \mathrm{r}^{\circ}$.

${ }^{11}$ Berthoin-Mathieu 1996 : $140 ; 162 ; 166 ; 178 ; 204 ; 218$; Ohrt 1921 : n 924.

${ }^{12}$ Lecouteux $2005: \mathrm{n}^{\circ} 278$ et 284 ; Storms $1948: \mathrm{n}^{\circ} 27$; Berthoin-Mathieu $1996: 62$; 130 ; Braekman $1997: \mathrm{n}^{\circ} 78$.

${ }^{13}$ Deux rédactions chez Aymar 1926 : 331 et 339 ; une autre British Library, ms. Sloane 2584 , fol. $45 \mathrm{v}^{\circ}$.

${ }^{14}$ Marcelli de medicamentis (éd. Niedermann 1916) ; Textes latins et vieux français relatifs aux Cyranides (éd. Delatte 1942); Picatrix (éd. Pingree 1986). 
${ }^{15}$ Par exemple chez Frommann 1675.

${ }^{16}$ Cockayne, in : Leechdoms, Wortcunning and Starcraft in Early England.

${ }^{17}$ VRSVSMV / SMQLIVB / CSSML / NDSMD. Villiers 1989 : 81 sq. ; Franz 1909 : II, 107.

${ }^{18}$ Scott 1584 : XII, 18 ; Braekman $1997: 147 ; 12 ; 13 ; 18 ; 164$; Ohrt 1921 : n 173 ; Dragon noir : 165 ; Enchiridion Leonis papae, Ancône, 1667 : 104 ; Thiers I, 377 ; 413. Ohrt $1921: \mathrm{n}^{\circ} 267$; van Haver $1964: \mathrm{n}^{\circ} 682$.

19 " Au nom du Père, du Fils et du Saint Esprit » s'abrège ainsi en allemand : I.N.G.d.V.d.S.u.d.hl.G. ; dans les charmes néerlandais, W.g. (West gegroet) est le début de la Salutation angélique.

${ }^{20}$ Marcellus : chap. 10 ; Heim $1893: 491$.

${ }^{21}$ Reproduit chez Blaise de Vigenère 1587.

${ }^{22}$ Reproduit chez Lecouteux 1996 : 22.

${ }^{23}$ Reproduit chez Blaise de Vigenère 1587.

${ }^{24}$ Comme : oGooeee lo ce 9 q vo766 IGIG 66 (Hervé $\left.1916: 364\right) ; \mathrm{X} \mathrm{M} \mathrm{O} \Theta \mathrm{X}$ A $\rho \dagger \dagger \varepsilon \mathrm{A}$ $4 \lambda$ a L x z v 8 V v (Ohrt 1921 : II, 130).

${ }^{25}$ Wasserschleben $1851: 272 ; 335 ; 356 ; 480$ passim.

${ }^{26}$ Cf. Pourrat 2009 : $417 ; 422 ; 661 ; 1199$.

${ }^{27}$ Dans la mythologie germanique, le dragon Fafnir est le possesseur du heaume de terreur.

\section{RÉFÉRENCES}

Albertus Magnus Ägyptische Geheimnisse, bewährte und approbirte sympathetische und natürliche egyptische Geheimnisse für Menschen und Vieh, 4 fascicules, Braband, s.d., 1, 222.

Arnaldus de Villanova, Opera, Lyon, 1509.

Árnason, Jón 1862-1864. Islenzkar bióðsögur og aefintyri, 2 vol. Leipzig: J.C. Hinrichs. Ars notoria. The Notory Art of Solomon shewing the Cabanistical Key of magical Operations... Londres: J. Cottrel, 1657.

Aymar, Alphonse 1926. Le sachet accoucheur et ses mystères. Contribution à l'étude du folklore de la Haute-Auvergne du XIII ${ }^{\mathrm{e}}$ au XVIII ${ }^{\mathrm{e}}$ siècles. Annales du Midi $38,273-347$.

Bang, A. Chr. 2005. Norske hexeformularer og magiske opskrifter. Oslo: Kildeforlaget. (1ère édition Kristiania, 1901-1902 [Videnskabsselskabets skrifter 2. Historiskfilos. klasse $\left.1901, \mathrm{n}^{\circ} 1\right]$ ).

Beckers, Hartmut 1984. Eine spätmittelalterliche deutsche Anleitung zur Teufelsbeschwörung mit Runenschriftverwendung. Zeitschrift für deutsches Altertum 113, 136-145. 
Berthoin-Mathieu, Anne 1996. Prescriptions magiques anglaises du X $X^{e}$ au XII ${ }^{e}$ siècle, 2 vol. Paris: Publications de l'Association des Médiévistes Anglicistes.

Blaise de Vigenère 1587. Traicté des chiffres ou secrètes manières d'escrire. Paris: chez Abel L'Angelier.

Bohak, Gideon 2003. Hebrew, Hebrew everywhere? Notes on the Interpretation of Voces magicae, in S. Noegel, J. Walker, Br. Wheeler (éd.), Prayer, Magic and the Stars in the ancient and late Antique World. Philadelphie: Pennsylvania University Press, 69-82.

Bonner, Campbell 1950. Studies in Magical Amulets chiefly Graeco-Egyptian. Ann Arbor: Chicago University Press.

Boswinkel, Ernst, Pestman, Pieter W. (éd.) 1978. Textes grecs, démotiques et bilingues. Leyde: E. J. Brill.

Braekman, Willy L. 1997. Middeleeuwse witte en zwart magie in het Nederlands taalgebied. Gecommentarieerd compendium van incantamenta tot einde $16^{\text {de }}$ eeuw. Gand: Koninklijke academie voor Nederlandse Taal- en Letterkunde.

Le Mesnagier de Paris 1994. Texte édité par G. E. Brereton et J. M. Ferrier, traduction et notes par K. Ueltschi. Paris: Librairie générale française.

Cockayne, T. O. 1864. Leechdoms, Wortcunning and Starcraft in Early England, 2 vol. London: Longman.

Delatte, Louis (éd.) 1942. Textes latins et vieux français relatifs aux Cyranides. Liège, Paris: Librairie Droz (Bibliothèque de philosophie et lettres de l'Université de Liège, XCIII)

Deonna, Waldemar 1944. Abra, Abraca. La croix-talisman de Lausanne. Genava 22, 116-137.

Espeland, V. 1974. Svartbok frå Gudbrandsdalen, Oslo, Bergen, Tromsø, Universitetsforlaget (Norsk Folkeminnelags Skrifter, 110).

Franz, Adolph 1909. Die kirchlichen Benediktionen im Mittelalter, 2 vol. Graz: Akademische Druck- u. Verlagsanstalt.

Frommann, J. Chr. 1675. Tractatus de fascinatione novus et singularis. Nuremberg: Joh. Andreae Endteri.

Gompert, L. 1918. Der Zachariassegen gegen die Pest. Hessische Blätter für Volkskunde $17,37-52$.

Gravier, Maurice (éd.) 1955. La Saga d'Eric le rouge. Paris: Aubier.

Haver, Jozef van 1964. Nederlanse Incantatieliteratuur. Een gecommentarieerd compendium van Nederlandse Besweringsformules. Gand: Secretariaat van de Koninklijke academie voor Nederlandse Taal- en Letterkunde.

Hedegård, Gösta 2002. Liber Iuratus Honorii: A Critical Edition of the Latin Version of the Sworn Book of Honorius. Stockholm: Almqvist \& Wiksell (Studia Latina Stockholmiensia 48).

Hervé, Georges 1916. Superstitions populaires suisses concernant les armes, le tir, la guerre, les blessures. Revue anthropologique 26, 351-365.

Hunt, Tony $1994^{2}$. Popular Medicine in $13^{\text {th }}$-Century England. Introduction and Texts. Cambridge: D.S. Brewer.

Lecouteux, Claude 2001. Agla, Sator. Quelques remarques sur les charmes médicaux du Moyen Âge. Nouvelle Plume: Revue d'Études mythologiques et symboliques $2,19-34$. 
Lecouteux, Claude 1996. Charmes, conjurations et bénédictions: lexique et formules. Paris: Champion. (Essais 17).

Lecouteux, Claude 2004. Le Livre des amulettes et talismans. Paris: Imago.

Lecouteux, Claude 2005. Le livre des grimoires: aspects de la magie au Moyen Âge. Paris: Imago (deuxième édition).

Heim, Richard 1893. Incantamenta magica graeca latina. Jahrbücher für classische Philologie 19, 463-576.

Niedermann M (éd.) 1916. Marcelli de medicamentis liber. Leipzig - Berlin: B.G. Teubener (Corpus medicorum latinorum, 5).

Ogden David 1999. Voces magicae, Letters, Shapes and Images, in V. Flint (éd.), Witchcraft and Magic in Europe: Ancient Greece and Rome. Londres: Barnes \& Noble.

Ohrt Ferdinand 1921. Danmarks trylleformler, 2 vol. Copenhague, Kristiana: Nordisk Forlag.

Peterson, E. 1926. Engel- und Dämonennamen: Nomina barbara. Rheinisches Museum N.F. 75, 393-421.

Pingree, David (éd.) 1986. Picatrix. The Latin Version of the Ghayat al-Hakîm. Londres: University of London (Studies of the Warburg Institute 39).

Pourrat, Henri 2009. Le trésor des contes. Paris: Robert Laffont.

Saemundsson, Matthiás Viðar 1992. Galdrar á Íslandi. Íslenzk galdrabók. Reykjavik: Almenna bókafélagið.

Scott, Reginald 1584. Discoverie of witchcraft. Londres.

Seligman, K. 1921. Ananisapta und Sator. Hessische Blätter für Volkskunde 20, 1-25.

Singer, Charles 1917. On a Greek charms used in England in the $12^{\text {th }}$ century. Annals of Medical History 1, 258-260.

Storms, G. 1948. Anglo-Saxon Magic. La Haye: Martinus.

Testament de Salomon, trad. dans Lecouteux 2005, 66-70.

Timotin, Emanuela 2009. Les charmes roumains manuscrits. Évolution et transmission d'un savoir traditionnel aux XVII ${ }^{e}-X I X^{e}$ siècles, thèse, Grenoble III.

Véronèse, Julien 2007. L“Ars notoria” au Moyen Âge. Florence, Sismel - Edizioni del Galluzzo.

Villiers, Elisabeth 1989. Amuleti, Talismani, ed altre cose misteriosi. Milan: Hoepli Editore.

Wackernagel, Wilhelm 1876. Altdeutsche Predigten und Gebete aus Handschriften. Bâle: Hugo Richter.

Wasserschleben, F. W. H. 1851. Die Bußordnungen der abendländischen Kirche. Halle: Graeger.

Wesche, Heinrich 1940. Der althochdeutsche Wortschatz im Gebiete des Zaubers und der Weissagung. Halle: Niemeyer.

\section{About the author}

Claude Lecouteux is Professor Emeritus of medieval literature and civilization at the Sorbonne (Paris IV). He is the author of numerous books on folk beliefs and magic. For further informations see: http://fr.wikipedia.org/wiki/ Claude_Lecouteux . 


\section{Incantatio \\ An International Journal on \\ Charms, Charmers and Charming \\ http://www.folklore.ee/incantatio \\ Print version \\ Issue 2 \\ 2012}


ISNFR Committee on Charms, Charmers and Charming

\section{Incantatio}

An International Journal on

Charms, Charmers and Charming

\section{Issue 2}

General Editor: Mare Kõiva

Guest Editor for This Issue: Emanuela Timotin

Tartu 2012 
General Editor: Mare Kõiva

Guest Editor for This Issue: Emanuela Timotin

Layout: Liisa Vesik

Editorial board:

Alaric Hall

Claude Lecouteux

Lea Olsan

Éva Pócs

Jonathan Roper

Emanuela Timotin

Andrey Toporkov

Daiva Vaitkevičienè

William F. Ryan

Editorial contacts:

http://www.foklore.ee/incantatio

incantatio@folklore.ee

Vanemuise 42, Tartu 51003, Estonia

Supported by and affiliated to projects SF0030181s08 and

EKKM09-159 of the Estonian Ministry of Education and Research, and the European Union through the European Regional Development Fund (Centre of Excellence in CECT).

All rights reserved.

Copyright 2012 the authors and the

ISFNR Committee on Charms, Charmers and Charming

ISSN 2228-1355

DOI 10.7592/Incantatio 


\section{Contents}

doi: 10.7592/Incantatio2012

From the power of words to the power of rhetoric: nonsense, pseudo-nonsense words, archaisms and artificially constructed compounds in Greek oral charms Haralampos Passalis doi: 10.7592/Incantatio2012_1_Passalis

Ivan the Terrible's Malady and Its Magical Cure

William F. Ryan

doi: 10.7592/Incantatio2012_1_Ryan

Typologie des formules magiques

Claude Lecouteux

doi: 10.7592/Incantatio2012_1_Lecouteux

Verbal Charms from a Seventeenth-Century Manuscript

Andrei Toporkov

doi: 10.7592/Incantatio2012_1_Toporkov

Maria Lactans and the Three Good Brothers

Eleonora Cianci

doi: 10.7592/Incantatio2012_1_Cianci

Termes albanais pour 'incantation'

Cătălina Vătăşescu

doi: 10.7592/Incantatio2012_1_Vatacescu

Get Dr Clague. Dr John Clague as Collector of Manx Charms

Stephen Miller

doi: 10.7592/Incantatio2012_1_Miller

Contemporary Charms and Charming in Adjara, Georgia

Jonathan Roper

doi: 10.7592/Incantatio2012_1_Roper 
doi: 10.7592/Incantatio2012_1_BookReview

James Alexander Kapaló, Text, Context and Performance. Gagauz Folk Religion in Discourse and Practice. Leiden-Boston: Brill, 2011, 352 pp. (Haralampos Passalis)

Т. А. Агапкина, А.Л. Топорков (ed.), Восточнославянские Заговоры: Аннотированный Библиографический Указазатель (East Slavic Charms: Annotated Bibliographical Index). Moscow: Пробел, 2011, 170 pp. (Svetlana Tsonkova)

T. A. Agapkina, Vostochnoslavianskie lechebnye zagovory v sravnitel'nom osveshchenii. Siuzhetika i obraz mira (East Slavic Healing Spells in a Comparative Light: Plot Structure and Image of the World), Moscow: Indrik 2010. 823 pp. (William F. Ryan)

A. L. Toporkov, Russkie zagovory iz rukopisnykh istochnikov XVIIpervoi poloviny XIX $v$. (Russian Manuscript Charms from 17th to First Half of the 19th Century). Moscow: Indrik 2010. 830 pp. 14 illustrations (William F. Ryan)

J. Roper (ed.), Charms, Charmers and Charming.

International Research on Verbal Magic. Palgrave Macmillan, 2009, 294 pp. (Emanuela Timotin)

Conference REPORT

doi: 10.7592/Incantatio2012_1_Reports

Oral Charms in Structural and Comparative Light. International Conference at the Russian State University for the Humanities and at the Russian Academy of Sciences (Moscow, 27-29 October 2011) (E. Kuznetsova, A. Toporkov) 